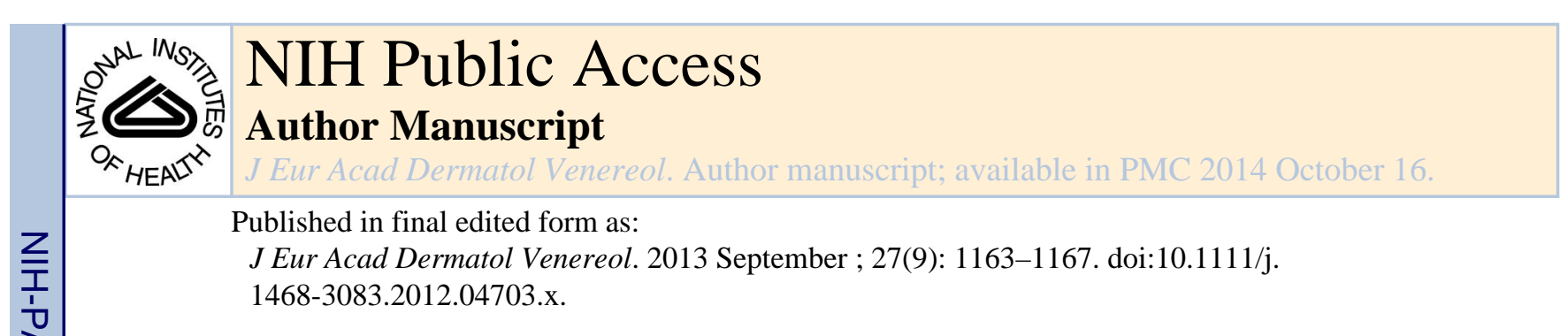

\title{
Depression and the risk of psoriasis in US women
}

\author{
PL Dominguez, MD1, J Han, PhD'1,2,3, T Li, MD, MS², A Ascherio, MD, DrPH²,3,4, and AA \\ Qureshi, MD, MPH ${ }^{1,2}$ \\ ${ }^{1}$ Department of Dermatology, Brigham and Women's Hospital and Harvard Medical School, \\ Boston, MA \\ ${ }^{2}$ Department of Channing Laboratory Department of Medicine, Brigham and Women's Hospital \\ and Harvard Medical School, Boston, MA \\ ${ }^{3}$ Department of Epidemiology, Harvard School of Public Health, Boston, MA \\ ${ }^{4}$ Department of Nutrition, Harvard School of Public Health, Boston, MA
}

\begin{abstract}
Background-Depression is a common mental health condition that has been associated with psoriasis. In the absence of prospective data, it is unclear whether depression precedes psoriasis as a risk factor.
\end{abstract}

Objective-To examine the association between depression and the risk of new-onset psoriasis.

Methods-A prospective cohort of 86880 US female nurses, The Nurses' Health Study II, was followed up from 1993 to 2005. Participants reported anti-depressant use and completed the Mental Health Index (MHI), a subscale of the Short-Form 36 in 1993. The MHI assessed for depression and scores were categorized into four strata: 0-52, 53-75, 76-85, and 86-100, with lower scores associated with increasing depressive symptoms. We excluded participants with a history of psoriasis prior to 1993. A self-report of incident physician-diagnosed psoriasis constituted the main outcome measure. For a sensitivity analysis, we had a subset of confirmed psoriasis cases.

Results-Depression was associated with an increased risk of incident psoriasis. Compared to women in the non-depressed group (MHI 86-100), women who reported either having high depressive symptomatology (MHI scores <52) or who were on anti-depressants had a multivariate RR of 1.59 for developing subsequent psoriasis (95\% confidence interval [CI], 1.21-2.08). These associations became stronger among confirmed psoriasis cases.

Conclusion-We found that depression was independently associated with an increased risk of psoriasis in this population of US women.

Corresponding Author: Abrar A. Qureshi, MD, MPH Assistant Professor, Harvard Medical School, Department of Dermatology, Brigham and Women's Hospital, 45 Francis St, 221L, Boston, MA 02115. Business phone: 617 525-7233, Fax: $617732-6373$, abrar.qureshi@channing.harvard.edu.

Declaration of Interest: Dr. Qureshi serves as a consultant to the Centers for Disease Control and Novartis. Drs. Dominguez, Han, Li, and Ascherio have no conflicts of interest to declare. The authors have not published or submitted any related papers from this study. 


\section{Introduction}

Psoriasis is a chronic inflammatory skin condition that affects 0.6 to $4.8 \%$ of the general population ${ }^{1}$. Depression is a common and sometimes debilitating condition that has become known to be associated with psoriasis ${ }^{2,3}$. For example, depression and depressive symptoms affect individuals with psoriasis disproportionately compared to individuals without psoriasis $^{4-6}$. Furthermore, the prevalence of depression and depressive symptomatology are higher amongst individuals with psoriasis 6,7 compared to individuals with other skin diseases such as lichen planus ${ }^{6}$, leprosy ${ }^{8}$, and vitiligo 9,10 . Reports have also shown that a depressed clinical state modulates itch perception in psoriasis ${ }^{11,12}$. In these reports, the severity of depression correlated directly with the severity of pruritus and an improvement in pruritus was associated with an improvement in depression scores among individuals with psoriasis ${ }^{12}$. Hence, it can be argued that that relationship between depression and psoriasis is bidirectional, psoriasis may affect depression, and depression may affect psoriasis.

Like psoriasis, substantial evidence now exists to support the notion that depression is an inflammatory condition with possible systemic consequences. For example, higher serum levels of inflammatory biomarkers such as IL-6 and CRP have been found in individual with depression compared to individuals without depression ${ }^{13}$, 14 . Depression is also well known to be associated with multiple conditions, such as psoriasis ${ }^{15}$, obesity ${ }^{16,17}$, coronary artery disease $^{18}$, in which inflammation is an underlying pathophysiological finding. This suggests that depression and psoriasis may be linked in part by shared pathways of inflammation.

Much of the data on psoriasis and depression derives from cross-sectional studies. In the absence of prospective data, it remains to be known if depression precedes psoriasis as a risk factor. In this study, we set out to examine the association between depression and the risk of psoriasis in individuals who self-reported a physician-diagnosis of psoriasis enrolled in a large US cohort of women.

\section{Method}

\section{Study population and assessment}

The Nurses' Health Study II (NHS II) is an ongoing longitudinal study of 116,608 female registered nurses from 15 US states who were between the ages of 25 and 42 years in 1989. The cohort is followed up with biennial questionnaires. The follow-up rate exceeds $90 \%$ for each 2-year period. In 2005, we asked participants if they had ever received a physician diagnosis of psoriasis and, if so, the date of the diagnosis. Participants were asked to indicate their diagnosis year in several intervals. For example, participants were asked if they received their psoriasis diagnosis before 1991 or between the years of 1991-1994, 1995-1998, 1999-2002, or after 2003. We confirmed psoriasis self-reports using the Psoriasis Screening Tool (PST) questionnaire ${ }^{19}$. The PST is a one page self-administered questionnaire that assigns a diagnosis of psoriasis based on responses to seven questions. The questions on the PST were derived from expert opinion and the National Psoriasis Foundation's survey on psoriasis. Three questions inquire about being diagnosed with psoriasis by some type of medical provider, such as a dermatologist, a primary care doctor, or a nurse practitioner. Four questions inquire about having morphological skin changes of 
psoriasis, three of which are accompanied by pictures. Pictures were chosen to represent each of three common psoriasis phenotypes, namely plaques, nail changes, and scalp psoriasis. One non-pictorial question inquires about inverse psoriasis. Scoring algorithms were developed to assign a diagnosis of psoriasis depending on a participant's response to the questions. These scoring algorithms were based on multiple a priori hypotheses. A pilot study showed that the PST has a $99 \%$ sensitivity and $94 \%$ specificity to detect psoriasis in a population of adults attending an outpatient dermatology clinic of a tertiary care referral center ${ }^{19}$. We mailed the PST to 2,430 NHS II participants who self-reported a physician diagnosis of psoriasis with an $87 \%$ response rate. Of these those who responded, $93 \%$ were confirmed to have psoriasis as determined by the PST.

In 1993, 1997, and 2001, we collected information on depressive symptoms. We assessed for depressive symptoms with the five-question Mental Health Index (MHI), a subscale of the Short-Form 36 health status surve ${ }^{20}$. The MHI includes five items designed to capture psychological distress versus well-being ${ }^{21}$. It asks respondents how much of the time over the past month (all, most, good bit, some, little, or none) they felt nervous, felt so down that nothing could cheer them up, felt calm and peaceful, felt down and blue, or felt happy. The scale is scored from 0 to 100 , with lower scores indicating higher levels of depressive symptomatology 22 . Based on a sample of patients diagnosed by clinical criteria, it was determined that a score of 52 or less on the MHI was highly predictive of clinical depression ${ }^{23}$. This score was used to define the "depressed" group. The MHI scale is not a diagnostic tool; it only identifies those with elevated depression-related symptoms. Participants were also classified as having depression if they reported ever taking fluoxetine, sertraline, or a tricyclic anti-depressant. Biennial follow-up questionnaires asked about smoking status and alcohol intake, and other lifestyle risk factors.

\section{Statistical analysis}

We used 1993 as baseline because the information on depression-related symptoms was collected first time this year. We included only the incident psoriasis cases diagnosed 1995 onwards. In the multivariate models, we adjusted for age (continuous), smoking status (never, current, or past smokers), alcohol intake (7 categories: none, 1-4 g/d, 5-9 g/d, 10-14 $\mathrm{g} / \mathrm{d}, 15-29 \mathrm{~g} / \mathrm{d}, 30-49 \mathrm{~g} / \mathrm{d}$, and $>50 \mathrm{~g} / \mathrm{d})$, and BMI $((<18.5,18.5-24.9,25-29.9,30-34.9$ and $35+)$. We computed person-time of follow-up for each participant from the return date of the 1993 questionnaire to the date of diagnosis of psoriasis or the return of 2005 questionnaire, whichever came first.

We used Cox proportional hazards models to estimate the multivariate relative risk (RR). We constructed interaction terms between the risk factors and calendar year and used likelihood ratio tests to assess the significance of these interaction terms, which suggested that the assumptions of proportional hazards were met. For all RRs, we calculated $95 \%$ confidence intervals (CIs). All $P$ values were 2-sided. All statistical analyses were performed using SAS software, version 9.1 (SAS Institute Inc, Cary, North Carolina).

The Partners Health Care System (Boston, Massachusetts) institutional review board approved this study. Return of a completed questionnaire was accepted by the institutional review board as implied informed consent. 


\section{Results}

\section{Basic characteristics}

The baseline characteristics of the cohort according to mental health score are shown in Table 1. In 1993, we had information on symptoms of depression in 86,880 women. Of these 86,880 participants, $85 \%$ reported to have some level of depressive symptomatology (MHI scores 0-85) (Table 1). Of these individuals who reported to have some level of depressive symptomatology, 5.4\% gave a self-report of having psoriasis. Towards higher levels of depression symptoms (lower MHI scores), participants were more likely to smoke, have a history of cancer, cardiovascular disease, type-2 diabetes, and take anti-depressant medication, and less likely to be married and be physically active.

\section{Depression and the risk of psoriasis}

The association between depression symptoms and the risk of subsequent development of psoriasis is presented in Table 2. According to the self-reported physician diagnosis of psoriasis 1995 onwards, depression measured at baseline 1993 was associated with an increased risk of psoriasis. These associations remained significant after adjusting for cigarette smoking, alcohol consumption, and BMI in the multivariate models. Women who were not on anti-depressants but who reported having some level of depressive symptoms (MHI scores 53-85) had a 45\% (MHI 76-85) and 57\% (MHI 53-75) increased risk of developing psoriasis compared to the non-depressed group (MHI 86-100). Women who were not on anti-depressants but who reported having the most severe depressive symptomatology (MHI < 52) had a RR of 1.59 (95\% CI, 1.21-2.08) compared to the nondepressed group. Women who reported both having severe depressive symptomatology and who reported anti-depressant use had a RR of 1.91 (95\% CI, 1.30-2.81) (Table 2). These associations are represented graphically in supplemental Fig. 1.

Depression's risk for psoriasis became stronger among confirmed psoriasis cases. For example, compared to the non-depressed group (MHI 86-100), women with the most severe depressive symptomatology (MHI <52) or who reported anti-depressant use had a RR of 1.68 (95\% CI, 1.13-2.47); furthermore, women with the most severe depressive symptomatology (MHI<52) and who reported anti-depressant use had a RR of 2.07 (95\% CI, 1.20-3.58) (Table 2). These associations are represented graphically in supplemental Fig. 2.

The analysis using the updated assessment of depressive symptoms yielded similar results. For example, compared with those with MHI of 86-100 and without anti-depression medication use, women with scores $<52$ or with medication use had a RR of 1.55 (95\% CI, 1.24-1.94); women with scores $<52$ and with medication use had a RR of 1.67 (95\% CI, 1.18-2.36). In addition, women on anti-depression medication alone had an increased risk of psoriasis compared with those without medication use (RR, 1.36; 95\% CI, 1.12-1.66).

\section{Discussion}

We observed that women who reported having depressive symptoms had an increased risk of developing psoriasis. The risk for psoriasis became more pronounced among those who 
were also on an anti-depressant medication. These associations became stronger in a subset of confirmed psoriasis self-reports. Previous cross-sectional studies have examined the association between depression and psoriasis. However in the absence of longitudinal data, it remains difficult to determine the temporal association between these two conditions. In one study, Gupta et. al. reported prospectively that a change in depression scores correlated with the change in pruritus in a group of inpatients. However these inpatients were already diagnosed with psoriasis ${ }^{12}$. In another study, Devrimci-Ozguven et. al. found that the risk for developing psoriasis increased significantly in patients with moderate and severe depression. However the ascertainment of depression occurred retrospectively ${ }^{5}$. To our knowledge, our study is the first to examine the association between depressive symptoms and prospective incidence of psoriasis.

This investigation was guided by a priori attention to potential biological mechanisms linking depression and incident psoriasis. The inflammation associated with psoriasis likely has systemic consequences given the co-morbid association with other systemic inflammatory conditions, such as psoriatic arthritis, obesity, and coronary artery disease. Recent data has shown that depression is also associated with systemic inflammation.

For example, studies have reported a positive correlation between depressed mood and circulating markers of inflammation such as IL-6 and C-reactive protein ${ }^{13}, 14$, which are proinflammatory cytokines and acute phase reactants seen in psoriasis. Serum levels of leptin, an adipose tissue derived hormone, have been found to be elevated in individuals with psoriasis ${ }^{24}$. Leptin is known to act as a pro-inflammatory cytokine through its ability to activate T-cells and increase levels of TNF-alpha and IL- $6^{25}$. Leptin may also play a role in depression. For example, leptin resistance has been shown to increase vulnerability to depression $^{26}$; furthermore, administration of leptin has also been shown to have antidepressant effects ${ }^{27}$. However these effects may not be observed in those who are leptin resistant, such as the obese. Obese individuals have high levels of leptin, which is thought to be due to leptin resistance, much like high insulin levels in diabetes mellitus type 2 are a consequence of resistance to insulin ${ }^{25}$. Such high levels of leptin in the obese may contribute to the chronic inflammation seen in obesity, which is prevalent in both depression ${ }^{17}$ and psoriasis ${ }^{15}$. This data suggests that depression could be an immunologically-mediated inflammatory condition, sharing inflammatory pathways with psoriasis $^{3}$.

The strengths of this study include prospective analysis, a large study population with adequate power, extensive data on risk factors collected prior to psoriasis ascertainment, validation of psoriasis cases, and a standardized measure of depressive symptoms.

Limitations of our study also deserve comment. The retrospective recall of psoriasis onset may have led to misclassification of psoriasis onset. This well-educated female cohort provides high quality data with little loss to follow-up but does not represent a random sample of US women. As in other epidemiological studies on psoriasis ${ }^{28-32}$, our diagnosis of psoriasis relied on self-reports of physician-diagnosed psoriasis and we did not require an examination by a dermatologist. Previous validation studies in the Nurses' Health Study for another skin condition, basal cell carcinoma, found self-reports to be greater than $90 \%$ accurate ${ }^{33,34}$. Furthermore we confirmed psoriasis cases using the PST $^{19}$, and of these 
those who responded, $93 \%$ were actually confirmed to have psoriasis as determined by the PST. We found a stronger association between risk of incident psoriasis and depressive symptoms. Depression was also self-reported and therefore subject to reporting bias. We included anti-depressant medication use as a surrogate for being depressed in addition to the self-reported MHI scores. Depression is a condition that can remit and recur, however our analysis did include updated data on depression.

In summary, we observed that symptoms of depression in women were related to an elevated risk of incident psoriasis. Future research is needed to confirm these findings and explore possible pathophysiologic mechanisms.

\section{Supplementary Material}

Refer to Web version on PubMed Central for supplementary material.

\section{Acknowledgments} We are indebted to the participants in the Nurses' Health Study II for their dedication and commitment. Dr. Qureshi
serves as a consultant to the Centers for Disease Control and Novartis. Drs. Dominguez, Han, Li, and Ascherio
have no conflicts of interest to declare. Dr. Han had full access to all of the data in the study and takes responsibility
for the integrity of the data and the accuracy of the data analysis.

Funding support: This study was supported in part by a grant from the Department of Dermatology, Brigham and Women's Hospital to AAQ. The NHSII cohort follow-up is funded by NIH CA50385.

\section{References}

1. Naldi L. Epidemiology of psoriasis. Curr Drug Targets Inflamm Allergy. Jun; 2004 3(2):121-128. [PubMed: 15180464]

2. Kessler RC, Nelson CB, McGonagle KA, Liu J, Swartz M, Blazer DG. Comorbidity of DSM-III-R major depressive disorder in the general population: results from the US National Comorbidity Survey. Br J Psychiatry Suppl. Jun.1996 (30):17-30. [PubMed: 8864145]

3. Filakovic P, Biljan D, Petek A. Depression in dermatology: an integrative perspective. Psychiatr Danub. Sep; 2008 20(3):419-425. [PubMed: 18827775]

4. Kilic A, Gulec MY, Gul U, Gulec H. Temperament and character profile of patients with psoriasis. Journal of the European Academy of Dermatology \& Venereology. 2008; 22(5):537-542. [PubMed: 18331306]

5. Devrimci-Ozguven H, Kundakci TN, Kumbasar H, Boyvat A. The depression, anxiety, life satisfaction and affective expression levels in psoriasis patients. [see comment]. Journal of the European Academy of Dermatology \& Venereology. 2000; 14(4):267-271. [PubMed: 11204514]

6. Akay A, Pekcanlar A, Bozdag KE, Altintas L, Karaman A. Assessment of depression in subjects with psoriasis vulgaris and lichen planus. Journal of the European Academy of Dermatology \& Venereology. 2002; 16(4):347-352. [PubMed: 12224690]

7. Esposito M, Saraceno R, Giunta A, Maccarone M, Chimenti S. An Italian study on psoriasis and depression. Dermatology. 2006; 212(2):123-127. [PubMed: 16484818]

8. Bharath S, Shamasundar C, Raghuram R, Subbakrishna DK. Psychiatric morbidity in leprosy and psoriasis--a comparative study. Indian Journal of Leprosy. 1997; 69(4):341-346. [PubMed: 9474509]

9. Sharma N, Koranne RV, Singh RK. Psychiatric morbidity in psoriasis and vitiligo: a comparative study. Journal of Dermatology. 2001; 28(8):419-423. [PubMed: 11560158]

10. Mattoo SK, Handa S, Kaur I, Gupta N, Malhotra R. Psychiatric morbidity in vitiligo and psoriasis: a comparative study from India. Journal of Dermatology. 2001; 28(8):424-432. [PubMed: 11560159] 
11. Gupta MA, Gupta AK, Schork NJ, Ellis CN. Depression modulates pruritus perception: a study of pruritus in psoriasis, atopic dermatitis, and chronic idiopathic urticaria. Psychosomatic Medicine. 1994; 56(1):36-40. [PubMed: 8197313]

12. Gupta MA, Gupta AK, Kirkby S, et al. Pruritus in psoriasis. A prospective study of some psychiatric and dermatologic correlates. Archives of dermatology. Jul; 1988 124(7):1052-1057. [PubMed: 3389849]

13. Bremmer MA, Beekman AT, Deeg DJ, et al. Inflammatory markers in late-life depression: results from a population-based study. J Affect Disord. Mar; 2008 106(3):249-255. [PubMed: 17716746]

14. Ford DE, Erlinger TP. Depression and C-reactive protein in US adults: data from the Third National Health and Nutrition Examination Survey. Arch Intern Med. May 10; 2004 164(9):10101014. [PubMed: 15136311]

15. Gottlieb AB, Dann F. Comorbidities in patients with psoriasis. The American journal of medicine. Dec; 2009 122(12):1150 e1151-1159. [PubMed: 19958894]

16. de Wit L, Luppino F, van Straten A, Penninx B, Zitman F, Cuijpers P. Depression and obesity: a meta-analysis of community-based studies. Psychiatry research. Jul 30; 178(2):230-235. [PubMed: 20462641]

17. Luppino FS, de Wit LM, Bouvy PF, et al. Overweight, obesity, and depression: a systematic review and meta-analysis of longitudinal studies. Archives of general psychiatry. Mar; 67(3):220 229. [PubMed: 20194822]

18. Halaris A. Comorbidity between depression and cardiovascular disease. Int Angiol. Apr; 2009 28(2):92-99. [PubMed: 19367238]

19. Dominguez PL, Assarpour A, Kuo H, Holt EW, Tyler S, Qureshi AA. Development and pilottesting of a psoriasis screening tool. Br J Dermatol. Oct; 2009 161(4):778-784. [PubMed: 19566664]

20. Ware JE Jr, Sherbourne CD. The MOS 36-item short-form health survey (SF-36). I. Conceptual framework and item selection. Med Care. Jun; 1992 30(6):473-483. [PubMed: 1593914]

21. McHorney CA, Ware JE Jr, Raczek AE. The MOS 36-Item Short-Form Health Survey (SF-36): II. Psychometric and clinical tests of validity in measuring physical and mental health constructs. Med Care. Mar; 1993 31(3):247-263. [PubMed: 8450681]

22. Kroenke CH, Bennett GG, Fuchs C, et al. Depressive symptoms and prospective incidence of colorectal cancer in women. Am J Epidemiol. Nov 1; 2005 162(9):839-848. [PubMed: 16207809]

23. Berwick DM, Murphy JM, Goldman PA, Ware JE Jr, Barsky AJ, Weinstein MC. Performance of a five-item mental health screening test. Med Care. Feb; 1991 29(2):169-176. [PubMed: 1994148]

24. Chen YJ, Wu CY, Shen JL, et al. Psoriasis independently associated with hyperleptinemia contributing to metabolic syndrome. Archives of dermatology. Dec; 2008 144(12):1571-1575. [PubMed: 19075139]

25. Taylor VH, Macqueen GM. The Role of Adipokines in Understanding the Associations between Obesity and Depression. Journal of obesity (Online). 2010

26. Lu XY. The leptin hypothesis of depression: a potential link between mood disorders and obesity? Current opinion in pharmacology. Dec; 2007 7(6):648-652. [PubMed: 18032111]

27. Lu XY, Kim CS, Frazer A, Zhang W. Leptin: a potential novel antidepressant. Proceedings of the National Academy of Sciences of the United States of America. Jan 31; 2006 103(5):1593-1598. [PubMed: 16423896]

28. Gelfand JM, Stern RS, Nijsten T, et al. The prevalence of psoriasis in African Americans: results from a population-based study. Journal of the American Academy of Dermatology. 2005; 52(1): 23-26. [PubMed: 15627076]

29. Olsen AO, Grjibovski A, Magnus P, Tambs K, Harris JR. Psoriasis in Norway as observed in a population-based Norwegian twin panel. British Journal of Dermatology. 2005; 153(2):346-351. [PubMed: 16086747]

30. Stern RS, Nijsten T, Feldman SR, Margolis DJ, Rolstad T. Psoriasis is common, carries a substantial burden even when not extensive, and is associated with widespread treatment dissatisfaction. Journal of Investigative Dermatology Symposium Proceedings. 2004; 9(2):136139. 
31. Ferrandiz C, Bordas X, Garcia-Patos V, Puig S, Pujol R, Smandia A. Prevalence of psoriasis in Spain (Epiderma Project: phase I). [see comment]. Journal of the European Academy of Dermatology \& Venereology. 2001; 15(1):20-23. [PubMed: 11451315]

32. Saraceno R, Mannheimer R, Chimenti S. Regional distribution of psoriasis in Italy. Journal of the European Academy of Dermatology \& Venereology. 2008; 22(3):324-329. [PubMed: 18269600]

33. Colditz GA, Martin P, Stampfer MJ, et al. Validation of questionnaire information on risk factors and disease outcomes in a prospective cohort study of women. American Journal of Epidemiology. 1986; 123(5):894-900. [PubMed: 3962971]

34. Hunter DJ, Colditz GA, Stampfer MJ, Rosner B, Willett WC, Speizer FE. Diet and risk of basal cell carcinoma of the skin in a prospective cohort of women. Annals of Epidemiology. 1992; 2(3): 231-239. [PubMed: 1342273] 


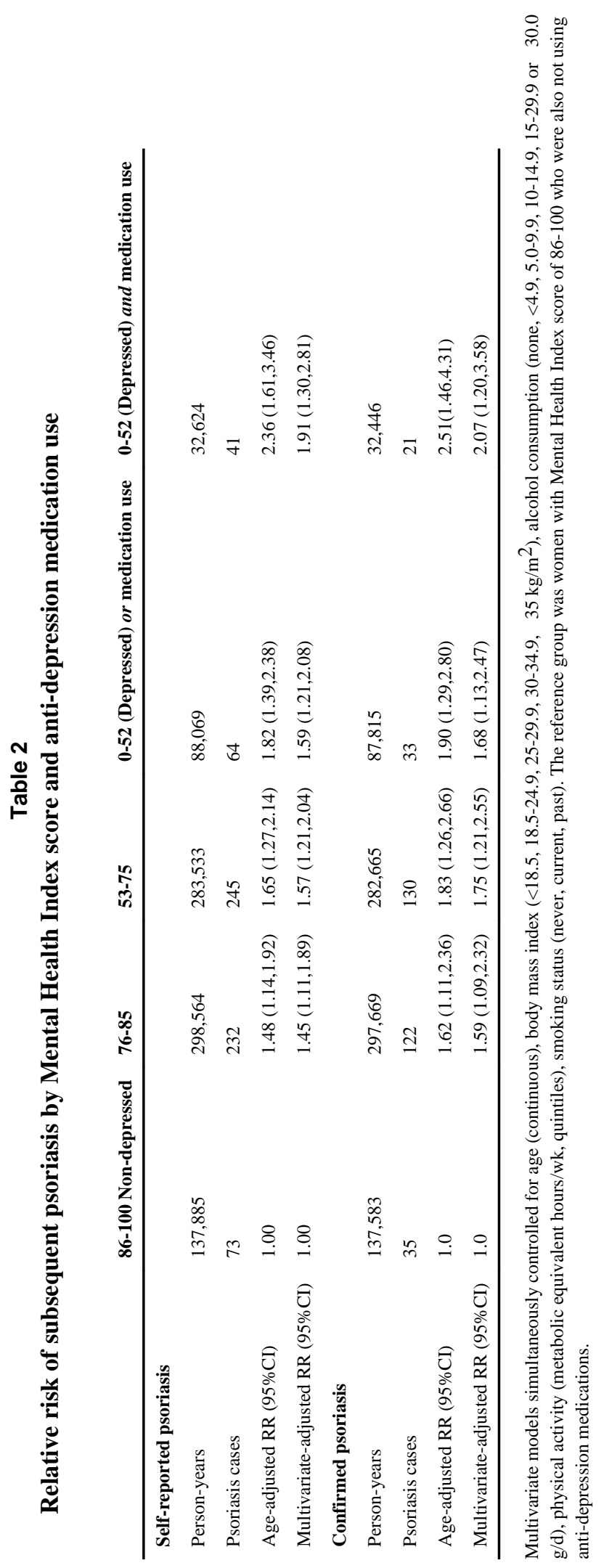

J Eur Acad Dermatol Venereol. Author manuscript; available in PMC 2014 October 16. 